\title{
Influence of Magnetic Field on Thermal Radiation and Particle Shapes of Copper-Water Nanofluid Considering Marangoni Boundary Layer
}

\author{
KM Kanika \\ Department of Mathematics, \\ Malaviya National Institute of Technology, Jaipur - 302017, Rajasthan, India. \\ Corresponding author: kanikatomar94@gmail.com \\ Santosh Chaudhary \\ Department of Mathematics, \\ Malaviya National Institute of Technology, Jaipur - 302017, Rajasthan, India. \\ E-mail: d11.santosh@yahoo.com \\ Mohan Kumar Choudhary \\ Department of Mathematics, \\ Muzaffarpur Institute of Technology, Muzaffarpur - 842003, Bihar, India. \\ E-mail: mkc2212@gmail.com
}

(Received January 29, 2019; Accepted April 11, 2020)

\begin{abstract}
This problem aims to address hydrodynamic Marangoni boundary layer flow of incompressible nanofluid along different shapes of particle like sphere, tetrahedron, column and lamina with exponential temperature. Choosing appropriate transformations, the governing equations are reduced to non-linear ordinary differential equations and then solved by using a perturbation technique. Impacts in velocity and temperature profiles for the relevant considering parameters namely nanoparticle volume fraction, magnetic parameter, empirical shape factor and radiation parameter are evaluated and shown through graphs. Moreover, computational values for influences of physical parameters on local surface heat flux are presented in table.
\end{abstract}

Keywords- Magnetic field, Thermal radiation, Nanofluid, Marangoni boundary layer.

\section{Introduction}

An analysis of electrically conducting fluids with magnetic field sources is named as Magnetohydrodynamic (MHD). Magnetic field can be applied to earn the liked nature of the industrial product via control the cooling rate. Therefore, the phenomenon of MHD flow has received great appreciation because of its applications in the branches of science and engineering. These applications involve bearings, plasma, crude oil purification, paper production, copper wire thinning, drawing and continuous strips cooling. The impact of magnetic field on fluid flow past a semi-infinite wall is developed initially by Rossow (1957). Although, Chaudhary and Kumar (2014) introduced the MHD boundary layer flow towards a flat plate and permeable surface. Latterly, some problems of fluid in the presence of magnetic field discussed by Chaudhary and Choudhary (2017), and Sharma et al. (2019).

Thermal radiation is a way of heat transmission from a system of higher temperature into a system of lower temperature with the condition that both systems are separated in space. The radiative heat transfer can be quite significant at high temperature and hence it can not be neglected. It has so many applications in the area of thermal engineering, chemical engineering, 
International Journal of Mathematical, Engineering and Management Sciences

Vol. 5, No. 5, 957-970, 2020

https://doi.org/10.33889/IJMEMS.2020.5.5.073

aeronautics and astrophysics, and geothermal reservoirs such as spacecraft reentry aerodynamics, gas turbines, comical flight aerodynamics rocket, nuclear power plants, polymer processing, glass production and reliable equipment. Influence of radiation models and radiative heat transfer on the modelling of hydrogen-hydrocarbon combustion is explored by Ilbas (2005). In addition, the influence of radiative heat flux on the boundary layer flow towards an exponential stretchable plate is introduced by Sajid and Hayat (2008). Moreover, several researchers like Jat and Chaudhary (2010), Wu and Cheng (2013), Chaudhary et al. (2015), Ramzan et al. (2017) and Chaudhary and Choudhary (2018) established the nature of thermal radiation along with distinct flow situations.

Conventional fluids namely engine oil, ethylene glycol, air, and toluene have minor thermal conductivity, while solid particles of gold $(\mathrm{Au})$, titanium dioxide $\left(\mathrm{TiO}_{2}\right)$, aluminum oxide $\left(\mathrm{Al}_{2} \mathrm{O}_{3}\right)$, copper oxide $(\mathrm{CuO})$, copper $(\mathrm{Cu})$ and silver $(\mathrm{Ag})$ have higher thermal conductivity. So to enhance the heat transfer rate of liquids with a significant augmentation of thermal conductivity, suspended the nano solid particles into the base fluid, this type of mixture is known as nanofluid. Consequently, nano-solid particles are taken typically with the size of diameter as 1 to $100 \mathrm{~nm}$. Examples of practical applications include heat exchangers, cancer therapy, thermal engineering and electronic chemical process. Notably, that applications are involved in heat sinks cooling, medical suspensions sterilization and powered engines. Choi and Eastman (1995) is pioneered the concept of nanofluid, in which he introduced an innovative way to increase advanced heat transfer by mixing nanoscaled particles into the ordinary fluid. However, some earlier published work on nanofluid illustrations are given by Bayat and Nikseresht (2012), and Sarafraz and Hormozi (2014). Further, Hajabdollahi and Hajabdollahi (2017), and Ahuja and Gupta (2019) are created the study of a few aspects like nanoparticle's size, shape and type in experimental or theoretical way.

Marangoni boundary layer can be created through liquid-gas or liquid-liquid interfaces. Marangoni convection has the appearance over to surface tension gradients, which may be both temperature and concentration gradient. Importance of Marangoni convection is carrying out by the feasibility of material processing in space craft, in this processing gravity force is taken as so small rather than the thermo-capillary one. Thus Marangoni effect have great attention in the applications of space processing and micro-gravity science like thin films spreading, processing of semi conductor, crystal growth melts, and nucleation vapor bubbles. Probably, Shivakumara et al. (1999) was the first one who pioneered the basic research work in the area of Marangoni boundary layer effect. Besides, some other studies have been carried out by Zhang and Zheng (2012), Gambaryan-Roisman (2015) and Aly and Ebaid (2016) with a Marangoni boundary layer under the different situations.

Keeping the discussed literature in view, the main goal of this article is to modulate the research problem of Lin et al. (2016) with the impact of a uniform transverse magnetic field on electrically conducting nanofluid. There are used solid nanoparticles as copper and base fluid as water containing different shapes of nanoparticles.

\section{Problem Description}

Let assume the Marangoni boundary layer flow of electrically conducting copper-water nanofluid along with the impact of radiation heat transfer and various particle shapes towards the temperature on surface $T_{w}=T_{\infty}+T_{0} e^{-X / L_{0}}$, where $T_{\infty}$ is the ambient fluid temperature, $T_{0}$ is the 
International Journal of Mathematical, Engineering and Management Sciences

Vol. 5, No. 5, 957-970, 2020

https://doi.org/10.33889/IJMEMS.2020.5.5.073

reference temperature and $L_{0}$ is the reference length. The flow is assumed steady, twodimensional and viscous. Although, $X$ and $Y$ are the Cartesian coordinates with the origin along and perpendicular to the surface, respectively and flow is bound at half plane $Y>0$. The solids and the liquid are taken in thermal equilibrium, and there is no slip condition between them. As presented in Figure 1, a variable magnetic field $B=B_{0} e^{-X / 3 L_{0}}$ is utilized in perpendicular direction of the flow, where $B_{0}$ is the magnitude of magnetic field strength. If the magnetic Reynolds number is surmised sufficiently small then caused magnetic field appears to be negligible. Until, the effect of Marangoni surface imitates as a boundary condition on the flow field governing equations. Further, thermophysical resources of water and copper are presented in Table 1, which is followed by Hayat et al. (2016).

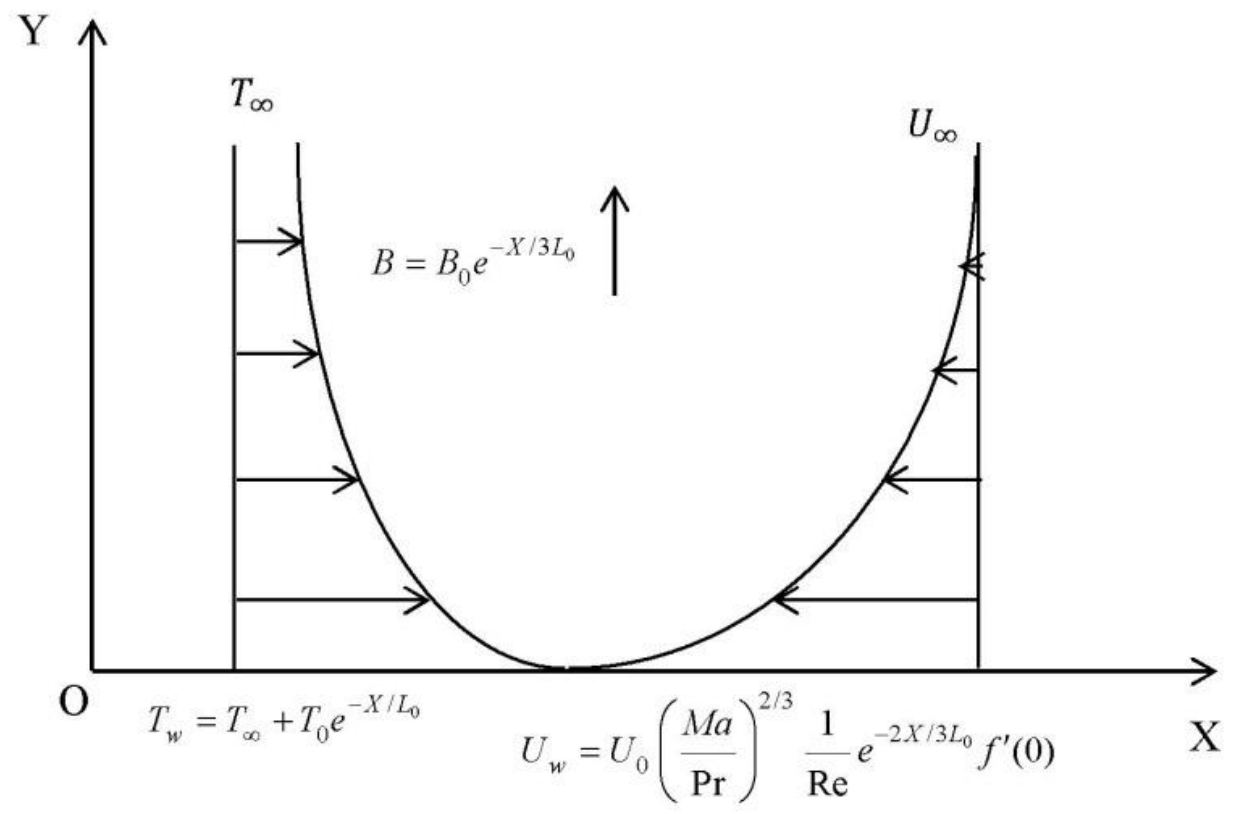

Figure 1. Geometry of the problem under consideration

Table 1. Thermophysical characteristics of used material

\begin{tabular}{|c|c|c|}
\hline Physical properties & Water & Copper \\
\hline$\kappa(W / m K)$ & 0.613 & 400 \\
\hline$\rho\left(K g / m^{3}\right)$ & 997.1 & 8933 \\
\hline$C_{p}(J / K g K)$ & 4179 & 385 \\
\hline$\sigma_{e}(S / m)$ & 0.05 & $5.96 \times 10^{7}$ \\
\hline
\end{tabular}


International Journal of Mathematical, Engineering and Management Sciences

Vol. 5, No. 5, 957-970, 2020

https://doi.org/10.33889/IJMEMS.2020.5.5.073

The basic equations under the approximations give

$\frac{\partial U}{\partial X}+\frac{\partial V}{\partial Y}=0$

$U \frac{\partial U}{\partial X}+V \frac{\partial U}{\partial Y}=v_{n f} \frac{\partial^{2} U}{\partial Y^{2}}-\frac{\left(\sigma_{e}\right)_{n f} B^{2}}{\rho_{n f}} U$

$U \frac{\partial T}{\partial X}+V \frac{\partial T}{\partial Y}=\alpha_{n f} \frac{\partial^{2} T}{\partial y^{2}}-\frac{1}{\left(\rho C_{p}\right)_{n f}} \frac{\partial q_{r}}{\partial Y}$.

the boundary conditions are expressed as

$\begin{array}{ll}\mu_{n f} \frac{\partial U}{\partial Y}=\frac{\partial \sigma}{\partial X}, V=0, T=T_{w} & \text { at } Y=0 \\ U \rightarrow 0, T \rightarrow T_{\infty} & \text { as } Y \rightarrow \infty .\end{array}$

where subscript $n f$ denotes the nanofluid's thermophysical properties, $U$ and $V$ are the velocity factors in the $X-$ and $Y$-axes, respectively, $v=\frac{\mu}{\rho}$ is the kinematic viscosity, $\mu$ is the viscosity coefficient, $\rho$ is the density, $\sigma_{e}$ is the electrical conductivity, $T$ is the nanofluid's temperature, $\alpha=\frac{\kappa}{\rho C_{p}}$ is the thermal diffusivity, $\kappa$ is the thermal conductivity, $C_{p}$ is the specific heat at constant pressure, $q_{r}=-\frac{4 \delta^{*}}{3 k^{*}} \frac{\partial T^{4}}{\partial Y}$ is the radiative heat flux, $\delta^{*}$ is the StefanBoltzmann constant, $k^{*}$ is the coefficient of mean absorption, $\sigma=\sigma_{0}-\gamma_{T}\left(T-T_{\infty}\right)$ is the tension at the surface, $\sigma_{0}$ is the positive constant and $\gamma_{T}$ is the temperature coefficient of the surface tension. It is considered that temperature difference inside the flow is very small like that $T^{4}$ can be signified as a linear function of $T_{\infty}$. Thus, expanding $T^{4}$ into the Taylor series about $T_{\infty}$, which after leaving higher order terms given the form $T^{4} \cong 4 T_{\infty}{ }^{3} T-3 T_{\infty}{ }^{4}$.

Followed by Ali et al. (2016), viscosity, density, electrical conductivity, thermal conductivity and heat capacity of the copper-water nanofluid are simplified as

$$
\begin{aligned}
\mu_{n f} & =\frac{\mu_{f}}{(1-\phi)^{2.5}} \\
\rho_{n f} & =\phi \rho_{s}+(1-\phi) \rho_{f}
\end{aligned}
$$


International Journal of Mathematical, Engineering and Management Sciences

Vol. 5, No. 5, 957-970, 2020

https://doi.org/10.33889/IJMEMS.2020.5.5.073

$$
\begin{aligned}
& \left(\sigma_{e}\right)_{n f}=\left(\sigma_{e}\right)_{f}+\frac{\left.3 \phi \mid\left(\sigma_{e}\right)_{s}-\left(\sigma_{e}\right)_{f}\right\rfloor}{\left(\sigma_{e}\right)_{s}+2\left(\sigma_{e}\right)_{f}-\phi\left[\left(\sigma_{e}\right)_{s}-\left(\sigma_{e}\right)_{f}\right]}\left(\sigma_{e}\right)_{f} \\
& \kappa_{n f}=\kappa_{f}+\frac{m \phi\left(\kappa_{s}-\kappa_{f}\right)}{\kappa_{s}+(m-1) \kappa_{f}-\phi\left(\kappa_{s}-\kappa_{f}\right)} \kappa_{f} \\
& \left(\rho C_{p}\right)_{n f}=\varphi\left(\rho C_{p}\right)_{s}+(1-\varphi)\left(\rho C_{p}\right)_{f} .
\end{aligned}
$$

where, subscript $s$ and $f$ indicate for copper particles and base fluid water, respectively, $\phi$ is the nanoparticle volume fraction, $m=\frac{3}{\varsigma}$ is the empirical shape factor and $\varsigma$ is the particles' sphericity. The proportion of the surface area of sphere to the surface area of particle along equal volumes is known as sphericity of the particle. Different shapes of the copper particles like sphere, tetrahedron, column and lamina are presented in Figure 2. Consequently, values of $\varsigma$ and $m$ for surmised shapes are shown in Table 2 given by Lin et al. (2016).

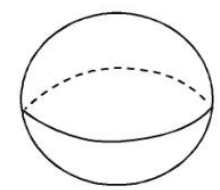

(i) Sphere

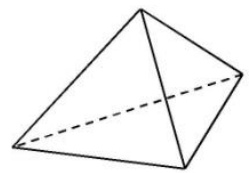

(ii) Tetrahedron

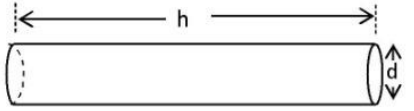

(iii) Column $d<h$

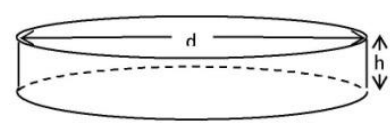

(iv) Lamina $d>h$

Figure 2. Shapes of nano solid copper particles

Table 2. Mathematical values of $\varsigma$ and $m$ for distinct nanoparticle shapes

\begin{tabular}{|c|c|c|}
\hline Model & $\varsigma$ & $m$ \\
\hline Sphere & 1 & 3 \\
\hline Tetrahedron & 0.7387 & 4.0613 \\
\hline Column & 0.4710 & 6.3698 \\
\hline Lamina & 0.1857 & 16.1576 \\
\hline
\end{tabular}

Introduce the following non-dimensional variables pursued by Lin et al. (2016) to convert the dimensional governing equations (1) to (3) into dimensionless form

$$
x=\frac{1}{L_{0}} X, \quad y=\frac{\sqrt{\mathrm{Re}}}{L_{0}} Y, \quad u=\frac{1}{U_{0}} U, \quad v=\frac{\sqrt{\mathrm{Re}}}{U_{0}} V, \quad t=\frac{1}{T_{\infty}} T .
$$

where $x$ and $y$ are dimensionless coordinates along the plate and perpendicular to it respectively, $u$ and $v$ are non-dimensional velocity along the $x$ - and $y$-axes respectively, 
International Journal of Mathematical, Engineering and Management Sciences

Vol. 5, No. 5, 957-970, 2020

https://doi.org/10.33889/IJMEMS.2020.5.5.073

$\operatorname{Re}=\frac{U_{0} L_{0}}{v_{f}}$ is the Reynolds number, $U_{0}$ is the reference velocity and $t$ is the dimensionless temperature. Thus the governing set equations (1) to (4) reduce to the following dimensionless form

$$
\begin{aligned}
& \frac{\partial u}{\partial x}+\frac{\partial v}{\partial y}=0 \\
& u \frac{\partial u}{\partial x}+v \frac{\partial u}{\partial y}=\frac{1}{(1-\phi)^{2.5}\left(1-\phi+\phi \frac{\rho_{s}}{\rho_{f}}\right)} \frac{\partial^{2} u}{\partial y^{2}}-\frac{\left(\sigma_{e}\right)_{n f} L_{0} B_{0}{ }^{2}}{\left(1-\phi+\phi \frac{\rho_{s}}{\rho_{f}}\right) U_{0} \rho_{f}} e^{-2 x / 3} u \\
& u \frac{\partial t}{\partial x}+v \frac{\partial t}{\partial y}=\frac{1}{\left[1-\varphi+\varphi \frac{\left(\rho C_{p}\right)_{s}}{\left(\rho C_{p}\right)_{f}}\right] \operatorname{Pr}}\left(\frac{\kappa_{n f}}{\kappa_{f}}+N r\right) \frac{\partial^{2} t}{\partial y^{2}} .
\end{aligned}
$$

subject to the transformed boundary conditions

$$
\begin{array}{ll}
\frac{T_{0}}{T_{\infty}} \frac{\partial u}{\partial y}=-(1-\varphi)^{2.5} \frac{M a}{\operatorname{Pr}}\left(\frac{1}{\operatorname{Re}}\right)^{3 / 2} & \frac{\partial t}{\partial x}, v=0, t=1+\frac{T_{0}}{T_{\infty}} e^{-x} \quad \text { at } y=0 . \\
u \rightarrow 0, t \rightarrow 1 & \text { as } y \rightarrow \infty
\end{array}
$$

where, $\operatorname{Pr}=\frac{v_{f}}{\alpha_{f}}$ is the Prandtl number, $N r=\frac{16 \delta^{*} T_{\infty}^{3}}{3 \kappa_{f} k^{*}}$ is the radiation parameter and $M a=\frac{\gamma_{T} T_{0} L_{0}}{\mu_{f} \alpha_{f}}$ is the Marangoni number.

\section{Transformation of Model}

Substituting the following dimensionless similarity variables (Lin et al. (2016)).

$\psi(x, y)=\left(\frac{M a}{\operatorname{Pr}}\right)^{1 / 3} \frac{1}{\sqrt{\operatorname{Re}}} e^{-x / 3} f(\eta), \quad \eta=\left(\frac{M a}{\operatorname{Pr}}\right)^{1 / 3} \frac{1}{\sqrt{\operatorname{Re}}} e^{-x / 3} y, \quad t(x, y)=1+\frac{T_{0}}{T_{\infty}} e^{-x} \theta(\eta)$.

where, $\psi(x, y)$ is the stream function defined as $u=\frac{\partial \psi}{\partial y}$ and $v=-\frac{\partial \psi}{\partial x}$, which automatically satisfies the continuity equation (11), $f(\eta)$ is the non-dimensional stream function, $\eta$ is the similarity variable and $\theta(\eta)$ is the non-dimensional temperature. Therefore, by using equation (15) in equations (12) to (14), obtain the following set of equations. 
International Journal of Mathematical, Engineering and Management Sciences

Vol. 5, No. 5, 957-970, 2020

https://doi.org/10.33889/IJMEMS.2020.5.5.073

$$
\begin{aligned}
& \frac{1}{(1-\phi)^{2.5}\left(1-\phi+\phi \frac{\rho_{s}}{\rho_{f}}\right)} f^{\prime \prime \prime}-\frac{1}{3} f f^{\prime \prime}+\frac{2}{3} f^{\prime 2}-\frac{M}{\left(1-\phi+\phi \frac{\rho_{s}}{\rho_{f}}\right)} \frac{\left(\sigma_{e}\right)_{n f}}{\left(\sigma_{e}\right)_{f}} f^{\prime}=0 \\
& \frac{1}{\left[1-\varphi+\varphi \frac{\left(\rho C_{p}\right)_{s}}{\left(\rho C_{p}\right)_{f}}\right] \operatorname{Pr}}\left(\frac{\kappa_{n f}}{\kappa_{f}}+N r\right) \theta^{\prime \prime}-\frac{1}{3} f \theta^{\prime}+f^{\prime} \theta=0 .
\end{aligned}
$$

with the corresponding boundary conditions

$$
\begin{aligned}
& f=0, f^{\prime \prime}=(1-\varphi)^{2.5}, \theta=1 \quad \text { at } \eta=0 \\
& f^{\prime} \rightarrow 0, \theta \rightarrow 0 \quad \text { as } \eta \rightarrow \infty .
\end{aligned}
$$

where prime denotes differentiation with respect to $\eta$ and $M=\frac{\left(\sigma_{e}\right)_{f} B_{0}{ }^{2} L_{0}(\operatorname{Pr})^{2 / 3} \operatorname{Re}}{\rho_{f} U_{0}(M a)^{2 / 3}}$ is the magnetic parameter.

The physical quantity is the local Nusselt number $\mathrm{Nu}$, which is described in dimensional form as

$$
N u=\frac{X\left(-\kappa_{n f} \frac{\partial T}{\partial Y}+q_{r}\right)_{Y=0}}{\kappa_{f}\left(T_{w}-T_{\infty}\right)}
$$

Using the above non-dimensional variables equation (15), the equation (19) is expressed by

$$
N u=-\left(\frac{M a}{\operatorname{Pr}}\right)^{1 / 3}\left(\frac{\kappa_{n f}}{\kappa_{f}}+N r\right) x e^{-x / 3} \theta^{\prime}(0) .
$$

\section{Solution by Perturbation Technique}

The solution set of equations (16) and (17) with the relevant boundary conditions equation (18) is found by using the perturbation technique. Utilize the power series in the term of the small magnetic parameter $M$ is considered as follows.

$f(\eta)=\sum_{i=0}^{\infty} M^{i} f_{i}(\eta), \quad \theta(\eta)=\sum_{j=0}^{\infty} M^{j} \theta_{j}(\eta)$

Substituting equation (21) and its derivative in the equations (16) to (18) and then equating the coefficients of like power of $M$ 
$\frac{1}{(1-\phi)^{2.5}\left(1-\phi+\phi \frac{\rho_{s}}{\rho_{f}}\right)} f_{0}^{\prime \prime \prime}-\frac{1}{3} f_{0} f_{0}^{\prime \prime}+\frac{2}{3} f_{0}^{\prime 2}=0$

$\frac{1}{\left[1-\phi+\phi \frac{\left(\rho C_{p}\right)_{s}}{\left(\rho C_{p}\right)_{f}}\right] \operatorname{Pr}}\left(\frac{\kappa_{n f}}{\kappa_{f}}+N r\right) \theta_{0}^{\prime \prime}-\frac{1}{3} f_{0} \theta_{0}^{\prime}+f_{0}^{\prime} \theta_{0}=0$

$\frac{1}{(1-\phi)^{2.5}\left(1-\phi+\phi \frac{\rho_{s}}{\rho_{f}}\right)} f_{1}^{\prime \prime \prime}-\frac{1}{3} f_{0} f_{1}^{\prime \prime}+\frac{4}{3} f_{0}^{\prime} f_{1}^{\prime}-\frac{1}{3} f_{0}^{\prime \prime} f_{1}=\frac{1}{\left(1-\phi+\phi \frac{\rho_{s}}{\rho_{f}}\right)} \frac{\left(\sigma_{e}\right)_{n f}}{\left(\sigma_{e}\right)_{f}} f_{0}^{\prime}$

$\frac{1}{\left[1-\phi+\phi \frac{\left(\rho C_{p}\right)_{s}}{\left(\rho C_{p}\right)_{f}}\right] \operatorname{Pr}}\left(\frac{\kappa_{n f}}{\kappa_{f}}+N r\right) \theta_{1}^{\prime \prime}-\frac{1}{3} f_{0} \theta_{1}^{\prime}+f_{0}^{\prime} \theta_{1}=\frac{1}{3} f_{1} \theta_{0}^{\prime}-f_{1}^{\prime} \theta_{0}$

$\frac{1}{(1-\phi)^{2.5}\left(1-\phi+\phi \frac{\rho_{s}}{\rho_{f}}\right)} f_{2}^{\prime \prime \prime}-\frac{1}{3} f_{0} f_{2}^{\prime \prime}+\frac{4}{3} f_{0}^{\prime} f_{2}^{\prime}-\frac{1}{3} f_{0}^{\prime \prime} f_{2}=\frac{1}{3} f_{1} f_{1}^{\prime \prime}-\frac{2}{3} f_{1}^{\prime 2}+\frac{1}{\left(1-\phi+\phi \frac{\rho_{s}}{\rho_{f}}\right)} \frac{\left(\sigma_{e}\right)_{n f}}{\left(\sigma_{e}\right)_{f}} f_{1}^{\prime}$

$\frac{1}{\left[1-\varphi+\varphi \frac{\left(\rho C_{p}\right)_{s}}{\left(\rho C_{p}\right)_{f}}\right] \operatorname{Pr}}\left(\frac{\kappa_{n f}}{\kappa_{f}}+N r\right) \theta_{2}^{\prime \prime}-\frac{1}{3} f_{0} \theta_{2}^{\prime}+f_{0}^{\prime} \theta_{2}=\frac{1}{3} f_{1} \theta_{1}^{\prime}-f_{1}^{\prime} \theta_{1}+\frac{1}{3} f_{2} \theta_{0}^{\prime}-f_{2}^{\prime} \theta_{0}$

the boundary conditions can be given as,

$$
\begin{aligned}
& f_{i}=0, \quad f_{0}^{\prime \prime}=(1-\varphi)^{2.5}, \quad f_{j}^{\prime \prime}=0, \quad \theta_{0}=1, \quad \theta_{j}=0 \quad \text { at } \eta=0 . \\
& f_{j}^{\prime} \rightarrow 0, \quad \theta_{i} \rightarrow 0 \quad i \geq 0, \quad j>0 \quad \text { as } \eta \rightarrow \infty
\end{aligned}
$$

Lin et al. (2016) were found out the equation (22) for the non-magnetic case. Applied the shooting technique with fourth order Runge-Kutta method for the computational results of the remaining ordinary differential equation, which is employed with step size 0.001 to attain the solution. The above procedure is replicated until the results correct up to the admissible accuracy of $10^{-7}$ level are revealed. 
International Journal of Mathematical, Engineering and Management Sciences

Vol. 5, No. 5, 957-970, 2020

https://doi.org/10.33889/IJMEMS.2020.5.5.073

\section{Discussion of the Results}

The purpose of this section is to illustrate the velocity $f^{\prime}(\eta)$ and temperature $\theta(\eta)$ distributions along with the different values of the nanoparticle volume fraction $\phi$, the magnetic parameter $M$, the empirical shape factor $m$ and the radiation parameter $N r$ through graphs. Moreover, numerical values for the effect of pertinent considering parameters on the surface heat flux $\theta^{\prime}(0)$ are also presented by table. At the time when finding the impact of anyone specified parameter on distributions, the another pertinent parameters are chosen a fixed number.

Figures 3 and 4 reveal the impact of $\phi$ and $M$ on $f^{\prime}(\eta)$ respectively. It is noticed that the velocity of fluid reduces as $\phi$ and $M$ increase.

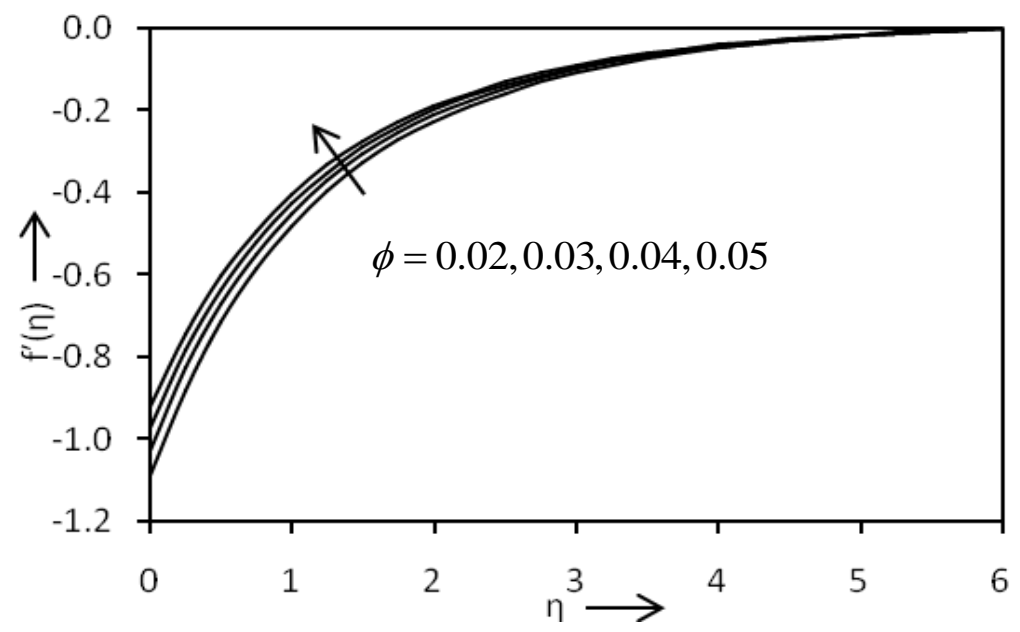

Figure 3. Variation of $\phi$ on the velocity profiles with $M=0.15$

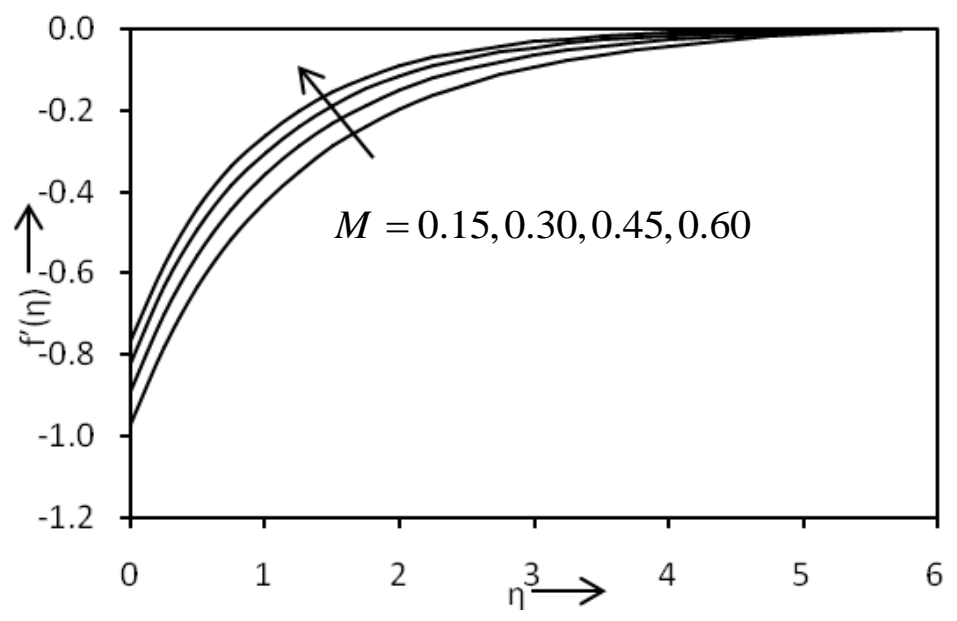

Figure 4. Variation of $M$ on the velocity profiles with $\phi=0.04$ 
International Journal of Mathematical, Engineering and Management Sciences

Vol. 5, No. 5, 957-970, 2020

https://doi.org/10.33889/IJMEMS.2020.5.5.073

Influence of $\phi, M, m$ and $N r$ on $\theta(\eta)$ are sketched in Figures 5 to 8 respectively. These figures display that enhancement in $\phi, M, N r$ implies to accretion in the thickness of thermal boundary layer. Apparently, It is also notable that for different shapes of nanoparticles particularly sphere, tetrahedron, column and lamina, fluid temperature step-up in the increasing order respectively.

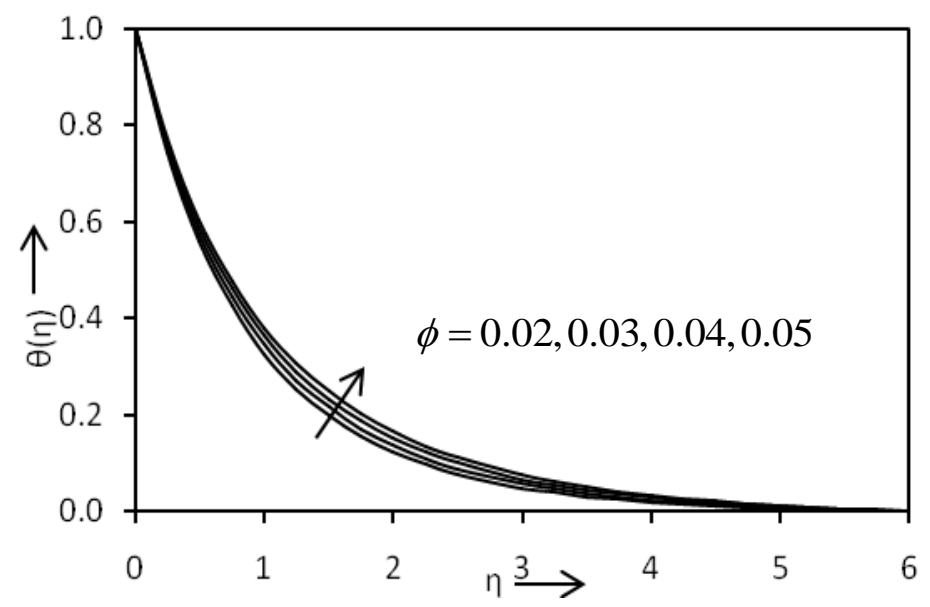

Figure 5. Variation of $\phi$ on the temperature profiles with $M=0.15, \operatorname{Pr}=6.8, N r=2$ and sphere nanoparticle

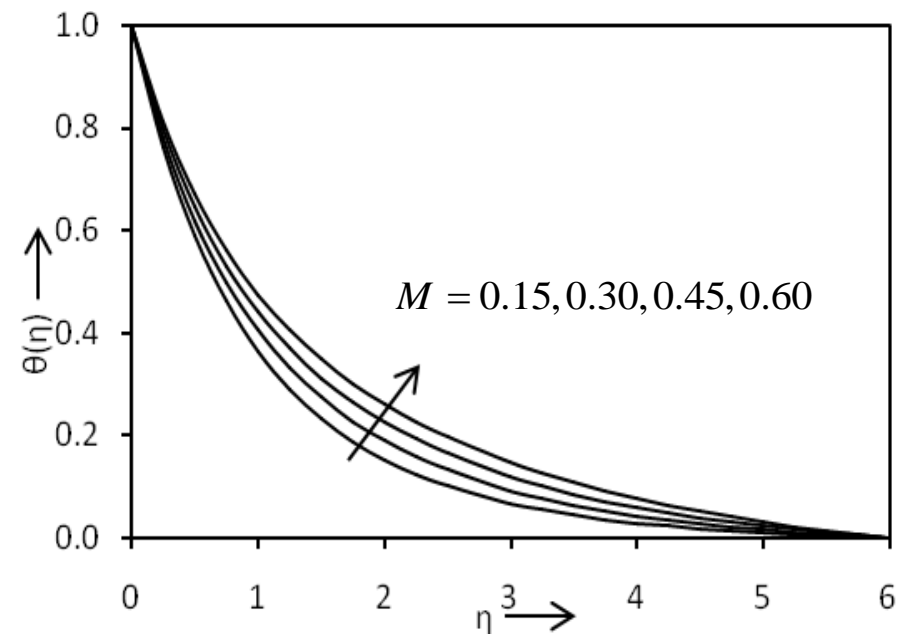

Figure 6. Variation of $M$ on the temperature profiles with $\phi=0.04, \operatorname{Pr}=6.8, N r=2$ and sphere nanoparticle 
International Journal of Mathematical, Engineering and Management Sciences

Vol. 5, No. 5, 957-970, 2020

https://doi.org/10.33889/IJMEMS.2020.5.5.073

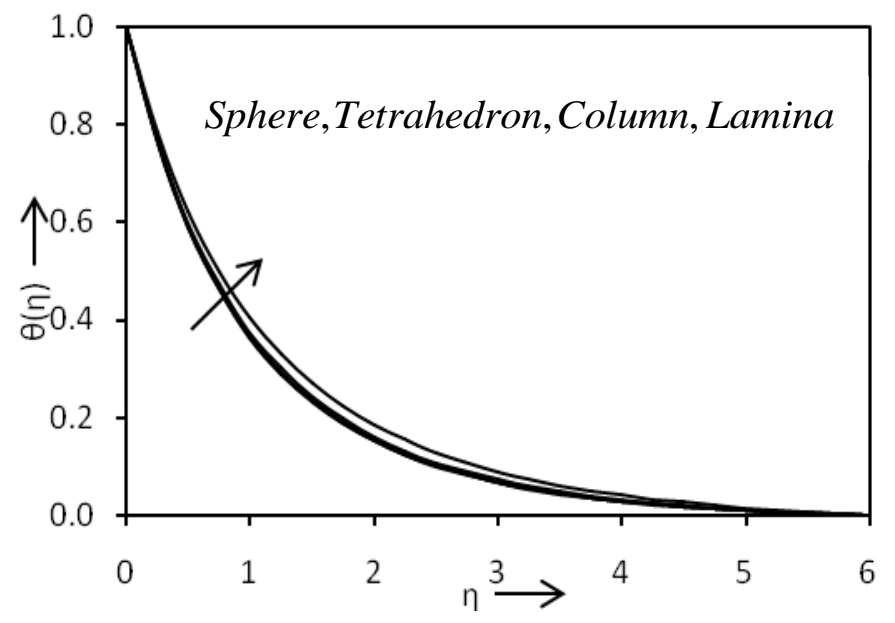

Figure 7. Variation of different shapes of solids on the temperature profiles with $\phi=0.04$,

$$
M=0.15, \operatorname{Pr}=6.8 \text { and } N r=2
$$

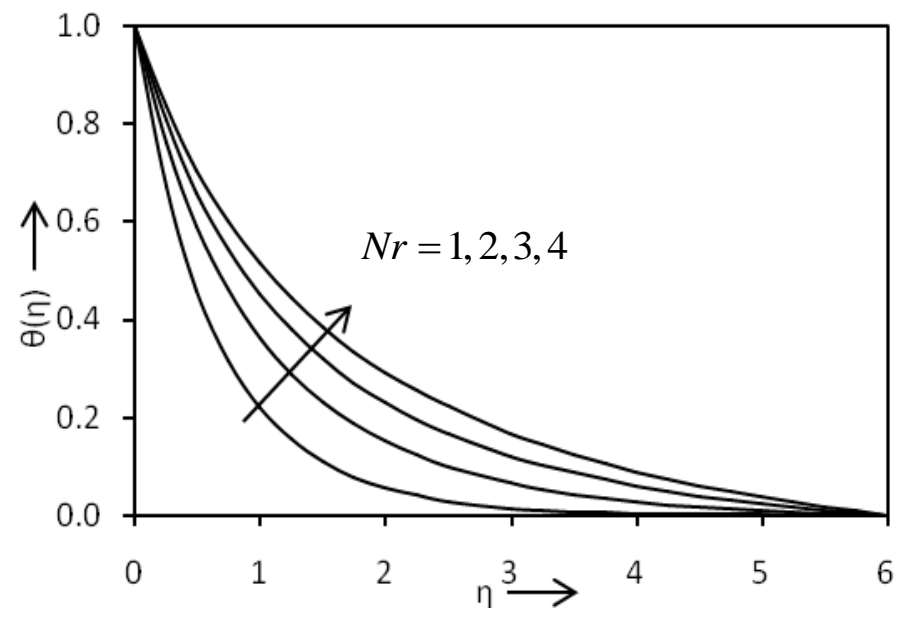

Figure 8. Variation of $\mathrm{Nr}$ on the temperature profiles with $\phi=0.04, M=0.15, \operatorname{Pr}=6.8$ and sphere nanoparticle

Table 3 illustrates the reflex of $\phi, M, m$ and $N r$ on $\theta^{\prime}(0)$. Further, $\theta^{\prime}(0)$ is proportional to the local Nusselt number $N u$. According to this table, $N u$ rises with the enhancing value of $\phi, M$, $m$ and $N r$. From the practical view point, negative sign of $\theta^{\prime}(0)$ denotes that there is heat transfer to the sheet. 
International Journal of Mathematical, Engineering and Management Sciences

Vol. 5, No. 5, 957-970, 2020

https://doi.org/10.33889/IJMEMS.2020.5.5.073

Table 3. Numerical results of $\theta^{\prime}(0)$ for different values of relevant specified parameters when $\operatorname{Pr}=6.8$

\begin{tabular}{|c|c|c|c|c|}
\hline$\phi$ & $M$ & $m$ & $\mathrm{Nr}$ & $-\theta^{\prime}(0)$ \\
\hline 0.02 & 0.15 & 3.0000 & 2 & 1.270172 \\
\hline 0.03 & & & & 1.214023 \\
\hline 0.04 & & & & 1.165352 \\
\hline 0.05 & & & & 1.087834 \\
\hline \multirow[t]{9}{*}{0.04} & 0.30 & & & 1.153103 \\
\hline & 0.45 & & & 1.134362 \\
\hline & 0.60 & & & 0.968817 \\
\hline & 0.15 & 4.0613 & & 1.083542 \\
\hline & & 6.3698 & & 1.038163 \\
\hline & & 16.1576 & & 1.014332 \\
\hline & & 3.0000 & 1 & 1.821459 \\
\hline & & & 3 & 1.098938 \\
\hline & & & 4 & 0.792467 \\
\hline
\end{tabular}

\section{Conclusions}

In the present study, a numerical model for the effect of radiative heat transfer and exponential temperature on the MHD Marangoni convection flow of incompressible copper-water nanofluid along the different four types shape of nanoparticles is explored. By using the non-dimensional transformation, the governing partial differential equations for continuity, momentum and energy with the appropriate boundary conditions are remodeled into the ordinary differential equations. Numerical results of the appeared equations are established through the perturbation technique. According to the observation of present study, the fluid velocity decreases with the enhancement in the value of the nanoparticle volume fraction and the magnetic parameter, while opposite circumstance exists in temperature of the fluid and heat transfer rate for the enhancing value of the nanoparticle volume fraction, the magnetic parameter and the radiation parameter. Consequently, lamina shaped nanoparticles have high temperature and surface heat flux as compared to the other nanoparticle shapes.

\section{Conflict of Interest}

Authors declare that they have no conflict of interest.

\section{Acknowledgement}

The authors wish to express their sincere thanks to the learned referees for careful reading of the article and valuable comments.

\section{References}

Ahuja, J., \& Gupta, U. (2019). Rayleigh-Benard convection for nano-fluids for more realistic boundary conditions (Rigid-Free and Rigid-Rigid) using Darcy model. International Journal of Mathematical, Engineering and Management Sciences, 4(1), 139-156.

Ali, F., Gohar, M., \& Khan, I. (2016). MHD flow of water-based Brinkman type nanofluid over a vertical plate embedded in a porous medium with variable surface velocity, temperature and concentration. Journal of Molecular Liquids, 223, 412-419. 
International Journal of Mathematical, Engineering and Management Sciences

Vol. 5, No. 5, 957-970, 2020

https://doi.org/10.33889/IJMEMS.2020.5.5.073

Aly, E.H., \& Ebaid, A. (2016). Exact analysis for the effect of heat transfer on MHD and radiation Marangoni boundary layer nanofluid flow past a surface embedded in a porous medium. Journal of Molecular Liquids, 215, 625-639.

Bayat, J., \& Nikseresht, A.H. (2012). Thermal performance and pressure drop analysis of nanofluids in turbulent forced convective flows. International Journal of Thermal Sciences, 60, 236-243.

Chaudhary, S., \& Choudhary, M.K. (2017). Viscous dissipation and Joule heating effects on an unsteady magnetohydrodynamic flow over a linearly stretching permeable surface with uniform wall temperature. Indian Journal of Pure \& Applied Physics, 55(12), 864-872.

Chaudhary, S., \& Choudhary, M.K. (2018). Partial slip and thermal radiation effects on hydromagnetic flow over an exponentially stretching surface with suction or blowing. Thermal Science, 22(2), 797808.

Chaudhary, S., \& Kumar, P. (2014). MHD forced convection boundary layer flow with a flat plate and porous substrate. Meccanica, 49(1), 69-77.

Chaudhary, S., Choudhary, M.K., \& Sharma, R. (2015). Effects of thermal radiation on hydromagnetic flow over an unsteady stretching sheet embedded in a porous medium in the presence of heat source or sink. Meccanica, 50(8), 1977-1987.

Choi, S.U., \& Eastman, J.A. (1995). Enhancing thermal conductivity of fluids with nanoparticles (No.ANL/MSD/CP-84938; CONF-951135-29). Argonne National Lab., IL, USA.

Gambaryan-Roisman, T. (2015). Modulation of Marangoni convection in liquid films. Advances in Colloid and Interface Science, 222, 319-331.

Hajabdollahi, H., \& Hajabdollahi, Z. (2017). Numerical study on impact behavior of nanoparticle shapes on the performance improvement of shell and tube heat exchanger. Chemical Engineering Research and Design, 125, 449-460.

Hayat, T., Imtiaz, M., \& Alsaedi, A. (2016). Melting heat transfer in the MHD flow of $\mathrm{Cu}$-water nanofluid with viscous dissipation and Joule heating. Advanced Powder Technology, 27(4), 1301-1308.

Ilbas, M. (2005). The effect of thermal radiation and radiation models on hydrogen-hydrocarbon combustion modelling. International Journal of Hydrogen Energy, 30(10), 1113-1126.

Jat, R.N., \& Chaudhary, S. (2010). Radiation effects on the MHD flow near the stagnation point of a stretching sheet. Zeitschrift fur angewandte Mathematik und Physik, 61(6), 1151-1154.

Lin, Y., Li, B., Zheng, L., \& Chen, G. (2016). Particle shape and radiation effects on Marangoni boundary layer flow and heat transfer of copper-water nanofluid driven by an exponential temperature. Powder Technology, 301, 379-386.

Ramzan, M., Bilal, M., \& Chung, J.D. (2017). Effects of thermal and solutal stratification on jeffrey magneto-nanofluid along an inclined stretching cylinder with thermal radiation and heat generation/absorption. International Journal of Mechanical Sciences, 131-132, 317-324.

Rossow, V.J. (1957). On flow of electrically conducting fluids over a flat plate in the presence of a transverse magnetic field. National Advisory Committee for Aeronautics. Ames Aeronautical Lab.; Moffett Field, CA, United States, NACA Annual Report, 44(1), 489-508.

Sajid, M., \& Hayat, T. (2008). Influence of thermal radiation on the boundary layer flow due to an exponentially stretching sheet. International Communications in Heat and Mass Transfer, 35(3), 347356.

Sarafraz, M.M., \& Hormozi, F. (2014). Nucleate pool boiling heat transfer characteristics of dilute $\mathrm{Al}_{2} \mathrm{O}_{3}$ ethyleneglycol nanofluids. International Communications in Heat and Mass Transfer, 58, 96-104. 
International Journal of Mathematical, Engineering and Management Sciences

Vol. 5, No. 5, 957-970, 2020

https://doi.org/10.33889/IJMEMS.2020.5.5.073

Sharma, J., Gupta, U., \& Shukla, S. (2019). A revised model for magneto convection in binary nanofluids. International Journal of Mathematical, Engineering and Management Sciences, 4(1), 131-138.

Shivakumara, I.S., Venkatachalappa, M., \& Suma, S.P. (1999). Exact analysis of Marangoni convection with throughflow. Actamechanica, 136(1-2), 109-117.

Wu, J., \& Cheng, X. (2013). Generalized thermal resistance and its application to thermal radiation based on entransy theory. International Journal of Heat and Mass Transfer, 58(1-2), 374-381.

Zhang, Y., \& Zheng, L. (2012). Analysis of MHD thermosolutal Marangoni convection with the heat generation and a first-order chemical reaction. Chemical Engineering Science, 69(1), 449-455. 